\title{
Left- and Right-Handers May Unintentionally Apply Different Cognitive Strategies in Response to Situational Demands: Study Using the Block-Wise Proportion Congruency Effect
}

\author{
Kazuhito Yoshizaki* (D), Yurina Watanabe \\ Department of Psychology, Aichi Shukutoku University, Nagakute, Japan \\ Email: *yoshizak@asu.aasa.ac.jp
}

How to cite this paper: Yoshizaki, K. and Watanabe, Y. (2022) Left- and Right-Handers May Unintentionally Apply Different Cognitive Strategies in Response to Situational Demands: Study Using the BlockWise Proportion Congruency Effect. Journal of Behavioral and Brain Science, 12, 10-21.

https://doi.org/10.4236/jbbs.2022.121002

Received: November 12, 2021

Accepted: January 16, 2022

Published: January 19, 2022

Copyright $\odot 2022$ by author(s) and Scientific Research Publishing Inc. This work is licensed under the Creative Commons Attribution International License (CC BY 4.0).

http://creativecommons.org/licenses/by/4.0/

\begin{abstract}
We investigated the effects of handedness on developing latent cognitive response strategies according to situation variables. We used the findings of the block-wise Proportion Congruency (PC) effect in which the proportion of congruent trials in a given block modulates the compatibility effect in its block. The block-wise PC effect cannot be intentionally controlled. A Simon task with three different types of Proportion Congruency (PC) in a given block was used to measure the block-wise PC effects. This task was administered to 24 left-handed and 24 right-handed participants. The reaction time and error rates, consistent with previous studies, demonstrated that the block-wise PC effect was observed in left- and right-handers. Crucially, the block-wise PC effect was more pronounced in left- than right-handers, suggesting that left- and right-handers unintentionally apply different cognitive strategies in response to conflict resolution experiences.
\end{abstract}

\section{Keywords}

Cognitive Control, Handedness, Block-Wise Proportion Congruency (PC) Effect

\section{Introduction}

The dominant hand allows us to conduct daily life tasks more efficiently. This efficiency reflects underlying neurological differences [1]. However, to date, there is little evidence or consensus on the relationship between handedness, cognitive functions and cerebral dominance (empirical studies in language production, spatial processing and face processing [2] [3] [4], review [5] [6]), although recent stu- 
dies have provided some evidence for the relationship. Cognitive control is used to adjust our strategies based on environmental and situational factors to achieve the current goals. Two aspects of cognitive control have been proposed, and the balance between them is considered sensitive to individual differences [7] [8]. These aspects are stability, the ability to protect the current goal from disturbing information during task execution, and flexibility, which is the ability to respond sensitively to disturbing information and switch targets.

Serrien and O'Regan [9] conducted a cognitive task that required inhibition and switching with 21 right-handed and 21 left-handed adults, based on the idea that cognitive control depends on the balance between conflicting aspects: stability and flexibility. The results showed that right-handed participants excelled at blocking disturbing information efficiently, or maintaining stability, whereas left-handed participants excelled in task switching or maintaining flexibility. However, there is no unanimous agreement about these findings, especially regarding the left-handed advantage in task switching or flexibility [5] [10]. Moreover, there is little accumulated knowledge about this issue.

Serrien and O'Regan [9] gave participants explicit cues informing of a future trial's task and asked them to follow the cues and respond accurately. Specifically, the participants in the distractor-present/absent tasks were required to respond to digits of a specific color based on the instructions for each block while ignoring surrounding distractors. It was assumed that performance's differences between distractors-present and distractors-absent trials would reflect the strength of inhibition or stability. On the contrary, participants in flexibility assessment tasks performed identical tasks to the previous trial (repetition trials) in half of the trials and changed the task (switch trials) in the other half. The switching cost between repetition and switch trials was calculated to indicate flexibility. The indices measured in Serrien and O'Regan's [9] study tasks were products of the participants' intentions.

The purpose of this study was to examine the effect of handedness on cognitive control, especially implicitly applying situational cognitive strategies, by measuring the extent to which unintentional different cognitive strategies are applied in response to situational factors. We focused on task-context-dependent modulation of congruency effects in the stimulus-response compatibility paradigm [11].

The Flanker task [12], the Stroop task [13], and the Simon task [14] have congruent effects known as the Flanker effect, the Stroop effect, and the Simon effect, respectively. These effects evaluate cognitive control [15] because responses to targets in these tasks are better in congruent than incongruent trials, and the congruent effects highlight the impact of task-irrelevant information. For example, in the Simon task, viewers are required to identify a target when the target is presented on the left or right side of a monitor or identify task-relevant information while ignoring a target's location or task-irrelevant information. The response buttons are typically located on the left and right sides; therefore, the target presentation position (task-irrelevant information) and the button position for respond- 
ing to the target match in the congruent condition, which is not the case in the incongruent condition.

Attentional selection efficacy, i.e., visual selectivity, inferred from congruency effects are sensitive to a task's context as defined by a given block's proportion of congruent trials (e.g., [16] [17] [18] [19] review for [20]). This is known as the block-wise Proportion Congruency (PC) effect. Experiencing incongruent trials in laboratory settings reduces the congruency effect, and crucially, this behavioral modulation cannot be intentionally controlled [21] [22] [23]. However, researchers differ about the cause of the block-wise PC effect [21]. The attentional view is one account of this effect, suggesting that attention shifts toward identifying taskrelevant information when incongruent trials appear more frequently (e.g., [16] [20] [24]). In contrast, another account emphasizes learning and proposes that stimulus-response (S-R) contingencies are employed to forecast the response most frequently associated with a given stimulus (e.g., [25] [26] [27]). These two views are not mutually exclusive, and they synergistically may influence the block-wise PC effect.

Note that an expansive interpretation of the stability-flexibility view of handedness in cognitive control would suggest that the block-wise PC effect reflects the direction of stability-flexibility. In particular, the larger the block-wise PC effect, the stronger the tendency for flexibility, whereas the smaller the effect, the stronger the tendency for stability. Applying the findings of Serrien and O'Regan [9] to the latent application of different cognitive strategies in response to situational factors predicts that the block-wise PC effect would be more pronounced in left- than right-handers.

We administered the Simon task with different PCs to the right- and left-handed participants and measured the block-level PC effect to test these predictions. Specifically, we prepared three blocks with different congruent trial proportions: 75\%, $50 \%$, and $25 \%$. It was predicted that the Simon effect would increase as the frequency of congruency trials increased, and this tendency would be more pronounced in left-handers.

\section{Method}

\subsection{Participants}

Twenty-four right-handed ( 8 men; mean age $=21.7$ years, $S D=1.72$; mean handedness score $=9.58, S D=0.64)$ and 24 left-handed ( 5 men; mean age $=20.5$, $S D=2.86$; mean handedness score $=-7.71, S D=1.77)$ graduate $(n=8)$ and undergraduate $(n=40)$ students took part in this experiment. Handedness was evaluated using the Japanese version of the FLANDERS handedness questionnaire [28]. Participants in the experiment were selected from a pool of participants recruited in undergraduate and graduate classes of Aichi Shukutoku University. All participants reported normal or corrected-to-normal vision. They were paid $¥ 500$ (US\$4.55) for participation. Each participant signed an informed consent form according to the research protocol for human participants. This study was approved 
by the ethics committee of the Department of Psychology in Aichi Shukutoku University (No. 2017-01-r02).

\subsection{Experimental Design}

We manipulated three mixed variables. The first variable was a between-participant factor, comprising the handedness group consisting of left- and right-handers. The remaining two variables were within factors, comprising the Proportion Congruency (PC) factor consisting of three conditions (75\%, 50\%, and 25\%) and the compatibility factor consisting of congruent and incongruent conditions.

\subsection{Apparatus and Stimuli}

A desktop computer equipped with a 24-in TFT monitor (BenQ, L2411Z) having a refresh rate of $144 \mathrm{~Hz}$ was used to present the stimuli. Presentation timing, and response and response time recording was controlled using SuperLab version 5.05 software (Cedrus company, San Pedro, CA, USA). Participants' responses were recorded using a response box (Cedrus RB-530), which recorded the responses accurately within $1 \mathrm{~ms}$. A chin rest was used to maintain the distance between the eyes and the monitor.

All stimuli were presented on a monitor with a white background. The four target stimuli were circles with a diameter subtending a visual angle of $1.53^{\circ}$, drawn with a black line and painted in red $(255,0,0)$, green $(0,255,0)$, yellow $(255,255$, $0)$, or blue $(0,0,255)$, respectively. The fixation point (“+”) was a cross drawn with a black line subtending a visual angle of $0.38^{\circ}$.

\subsection{Procedure}

Participants were tested individually in a small, dimly lit room. They were given explicit instructions to continue staring at the central fixation point during the task. They were also explained about the correspondence between the colors and responding hands (described below). This explanation was repeated each time a new block was presented. The viewing distance from participants' eyes to the monitor was $45 \mathrm{~cm}$, maintained by the chin rest.

The trial schedule was as follows. First, $500 \mathrm{~ms}$ after a warning tone and the central fixation point were presented, one of four possible color circles was presented for $150 \mathrm{~ms}$ along with the fixation point, $3.57^{\circ}$ horizontally to the left or right of it. Participants were required to discriminate the color of the target circle as soon and as accurately as possible. Half of the participants were asked to press the buttons located on their left or right with their right index finger if the target circle was blue or yellow and with their left index finger if the target circle was red or green. The other half of the participants were assigned the opposite correspondence between colors and the responding hand. A buzzer presented immediate feedback if a response was incorrect. A millisecond timer started at the onset of the target and stopped at the response. Only trials between $150 \mathrm{~ms}$ and $1000 \mathrm{~ms}$ were recorded as reaction times. The subsequent trial started one se- 
cond after a response or no response for one second after presenting the target. Figure 1 shows an example of stimulus presentation and response buttons.
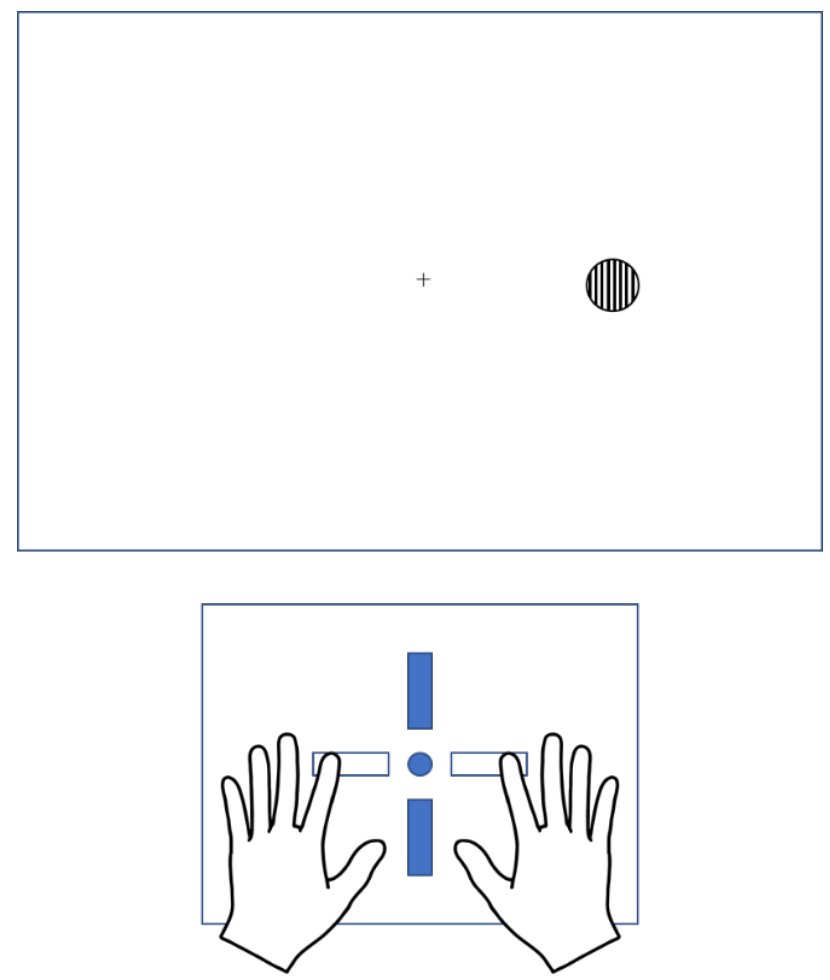

Figure 1. An example of stimulus presentation and response buttons in the Simon task. The target (the circle drawn by the vertical lines) is presented in a right visual field. The buttons used in this experiment are the ones located on the left and right. When the circle drawn by the vertical lines is red, then the red color corresponds to the left button (see text), which is an incongruent condition in this example.

Three block-types, each consisting of 64 trials having different proportions of congruent trials $(75 \%, 50 \%$, and $25 \%$ ), were presented. The four types of targets appeared 16 times in each block. Each participant responded to four consecutive blocks of each of the three types of blocks, or a total of 12 blocks (768 trials). A practice block of 32 trials having 50\% congruent trials preceded the experimental trials. The order of performing the three types of blocks was counterbalanced among the participants of each handedness group.

Participants reported whether they noticed a change in the proportion of congruent/incongruent trials by block after the experiment and what had changed if they did notice a change. No participants correctly reported a change.

\section{Results}

\subsection{Reaction Times}

Mean individual reaction times for correct responses were calculated for each participant. Figure 2 shows mean reaction times and standard errors in each experimental condition for left- and right-handers. 


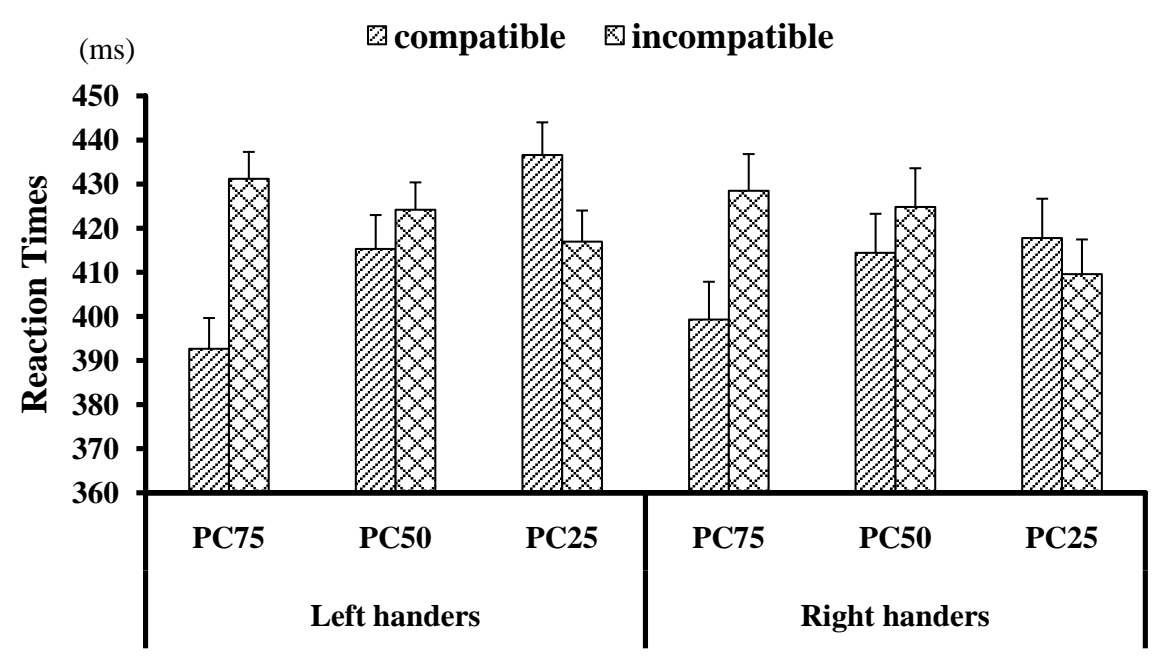

Figure 2. Mean reaction times for correct responses under each experimental condition in left- and right-handers. Error bars indicate standard errors. "PC75" indicates that the proportion congruent trials were $75 \%$ in a given block, similar to "PC50" and "PC25."

We conducted a three-way mixed analysis of variance (ANOVA) based on the experimental design, which indicated a significant main effect of compatibility, $F(1,46)=29.809, p<0.001, \quad \eta_{p}^{2}=0.393$, indicating a Simon effect $(9.9 \mathrm{~ms})$. None of the other main effects were significant (handedness, $F(1,46)=0.065, p=0.799$, $\eta_{p}^{2}=0.001$; PC, $\left.F(2,92)=2.300, p=0.106, \eta_{p}^{2}=0.048\right)$. There was a significant two-way interaction between PC and compatibility, $F(2,92)=103.737, p<0.001$, $\eta_{p}^{2}=0.693$, demonstrating a block-wise PC effect. Specifically, the Simon effect was larger in the PC75 condition (33.9 ms; $F(1,138)=165.475, p<0.001, \quad \eta_{p}^{2}=$ $0.545)$ than in the PC 50 condition $\left(9.6 \mathrm{~ms} ; F(1,138)=13.402, p<0.001, \eta_{p}^{2}=\right.$ 0.089), whereas a reverse Simon effect appeared in the PC25 condition $(-13.9 \mathrm{~ms}$; $\left.F(1,138)=27.973, p<0.001, \eta_{p}^{2}=0.169\right)$.

Crucially, the interaction between PC and compatibility differed according to handedness, $F(2,92)=4.927, p=0.009, \quad \eta_{p}^{2}=0.097$. Simple interactions in each handedness group showed that the block-wise PC effect was more pronounced in left-handers, $F(2,92)=76.937, p<0.001, \eta_{p}^{2}=0.626$, than right-handers, $F(2,92)$ $=31.728, p<0.001, \eta_{p}^{2}=0.408$. Specifically, as shown in Figure 2 the Simon effect was similarly large in both handedness groups for the $75 \%$ than in the $50 \%$ PC condition. In contrast, the reverse Simon effect was larger in left $(-19.7 \mathrm{~ms}$ : $\left.F(1,138)=27.874, p<0.001, \quad \eta_{p}^{2}=0.168\right)$ than right-handers ( $-8.2 \mathrm{~ms}: F(1,138)$ $=4.841, p=0.030, \eta_{p}^{2}=0.034$ ).

In order to examine the variation of the Simon effect according to the individual PC conditions of the participants by handedness, Figure 3 shows the Simon effect of all participants as a function of PC conditions. It was apparent that the Simon effect shifted more strongly in the negative direction as the PC became smaller for the left-handers than for the right-handers.

To indirectly confirm this trend, the difference in the Simon effect between the PC75 and PC25 conditions was calculated for each participant. Total mean 

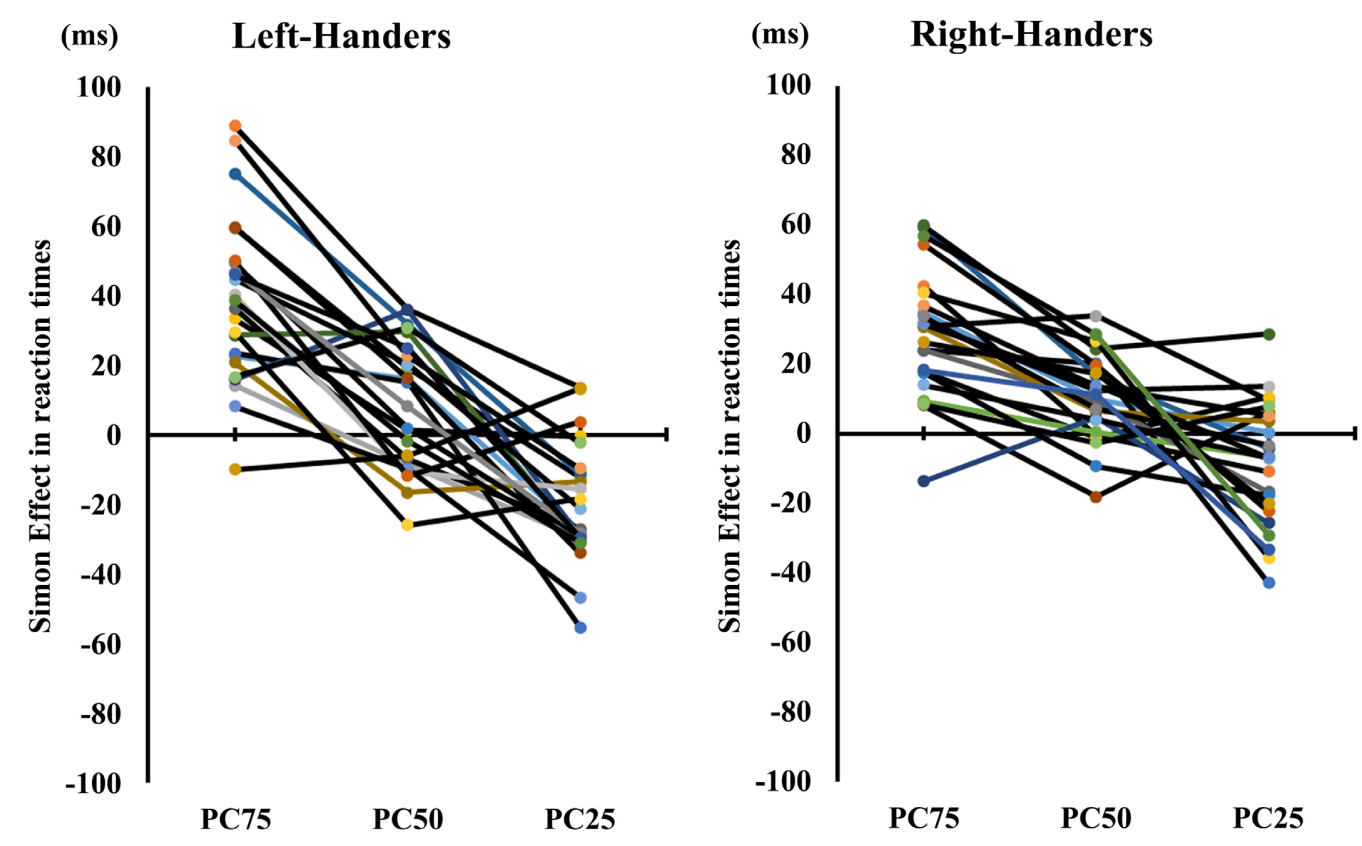

Figure 3. The Simon effect (reaction times) as a function of PC conditions in all participants for each handedness group. "PC75" indicates that the proportion congruent trials were $75 \%$ in a given block, similar to "PC50" and "PC25."

score for all participants $(n=48)$ was $47.80 \mathrm{~ms}(S D=26.63)$. The number of participants who indicated above the mean score was 17 out of 24 in the lefthanders, whereas it was 7 out of 24 in the right-handers, $\chi^{2}(1)=6.750, p<0.01$. These results were consistent with the results obtained from ANOVAs with reaction times as well as the trends that can be read from Figure 3.

\subsection{Error Rates}

Error rates in each experimental condition were calculated for each participant. Figure 4 shows mean error rates and standard errors in each experimental condition for left- and right-handers.

The error rates results were generally similar to reaction times results. A threeway mixed ANOVA was conducted on error rates. Similar to reaction time results, there was a two-way interaction between PC and compatibility, $F(2,92)=$ 53.788, $p<0.001, \eta_{p}^{2}=0.539$, indicating a block-wise PC effect. Importantly, this interaction differed according to handedness, $F(2,92)=5.974, p=0.004$, $\eta_{p}^{2}=0.115$. The simple interactions in each handedness group showed that the block-wise PC effect was larger in left, $F(2,92)=47.802, p<0.001, \eta_{p}^{2}=0.510$, than right-handers, $F(2,92)=11.960, p<0.001, \eta_{p}^{2}=0.206$. Moreover, as shown in Figure 4, the Simon effect in the PC75 condition was larger in left $\left(9.9 \%, F(1,138)=48.454, p<0.001, \eta_{p}^{2}=0.260\right)$ than right-handers $(4.7 \%$, $\left.F(1,138)=11.026, p=0.001, \eta_{p}^{2}=0.074\right)$. Furthermore, the reverse Simon effect in the PC25 condition was larger in left $(-7.5 \%, F(1,138)=27.775, p<0.001$, $\left.\eta_{p}^{2}=0.168\right)$ than right-handers $\left(-4.0 \%, F(1,138)=7.770, p=0.006, \eta_{p}^{2}=\right.$ $0.053)$. 


\section{$\square$ compatible $\bowtie$ incompatible}

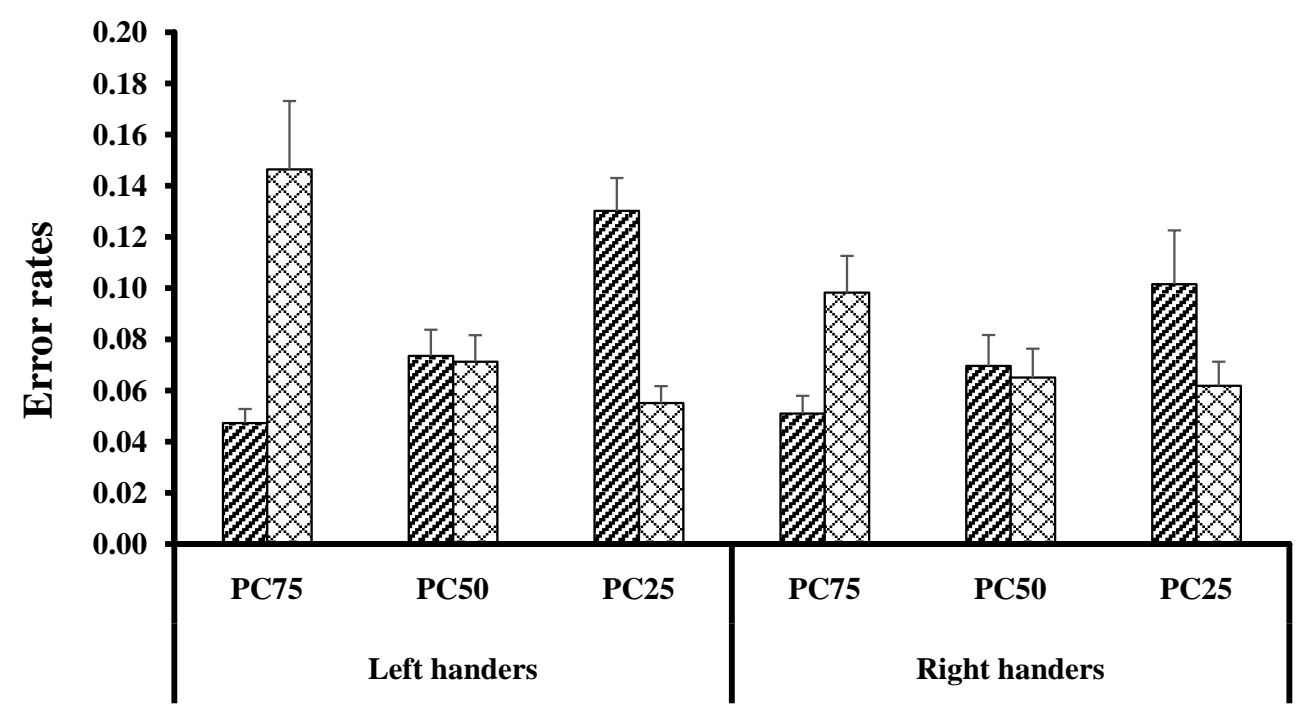

Figure 4. Mean error rates under each experimental condition in left- and right-handers. Error bars indicate standard errors. "PC75" indicates that proportion congruent trials were $75 \%$ in a given block, similar to "PC50" and "PC25."

\section{Discussion}

We investigated the effect of handedness on unintentional cognitive strategy application in response to situational demands by focusing on the Simon effect's modulation based on the block-wise proportion congruency (the block-wise PC effect). We assumed that the block-wise PC effect reflects the ease of unintentionally applying cognitive strategies based on the conflict frequency (incompatible trials) in a given block. We expanded the theory of handedness-based stability and flexibility of cognitive control [9] and predicted that the block-wise PC effect would be more prominent in left- than right-handers. This study's results supported this prediction.

This study provides initial evidence that left- and right-handers unintentionally apply different cognitive strategies in response to situational demands. The block-wise PC effect reflects "stability-flexibility" because experiencing conflicts and resolutions in a given block modulated cognitive strategies. In the present study, the block-wise PC effect obtained from reaction time and error rates was larger in left-handers than in right-handers, suggesting that left-handers are more sensitive to situational factors, i.e., experiences of conflicts and their resolutions. Conversely, right-handed people were better at maintaining independence from situational factors.

These findings enhance the generalizability of the stability-flexibility view of the handedness in cognitive control. As mentioned, Serrien and O'Regan [9] proposed this idea based on findings regarding situations in which participants conducted tasks under explicit instructions. In contrast, our experiment revealed that viewers unintentionally apply different cognitive strategies in response to their perception of a task situation, regardless of external instructions. We demonstrat- 
ed that the stability-flexibility view of handedness might also apply to situations where there is no external instruction.

However, a crucial question remains unanswered; what causes the unintentional application of different cognitive strategies depending on handedness in response to situational demands? One explanation might be the physiologically and functionally superior interhemispheric communication in left-handers [29] [30] [31]. Left-handers might have more task processing resources allowing them to develop cognitive strategies in response to a situation because of their superior interhemispheric interactions.

Another reason for the application of different cognitive strategies by handedness might be due to the degree of laterality. A recent functional magnetic resonance imaging study by Johnstone, Karlsson, and Carey [32] indicated that the hemispheric superiority for verbal information processing and the right-hemispheric superiority for facial and nonverbal information processing is less pronounced in left- than right-handed people, which cannot be explained by the right hemisphere's language dominance. These exciting findings suggest that left and right hemispheres' involvement in different functions is more bilateral in left- than right-handers. The increased bilateral involvement of left-handers might facilitate their access to ample processing resources from both hemispheres when performing a task, and the increased processing resources might result in unintentionally developing cognitive strategies.

We examined the Simon effect's visual field differences in left- and right-handed groups to identify if the increase in bi-hemispheric involvement of left-handers influenced the current study's results. Interestingly, a clear right visual field (VF) advantage for the Simon effect was observed in right-handers, (left VF $=-2.8 \mathrm{~ms}$ vs. right $\left.\mathrm{VF}=23.7 \mathrm{~ms}: F(1,46)=16.577, p<0.001, \eta_{p}^{2}=0.265\right)$. In contrast, no VF difference in the Simon effect was observed for left-handers (left VF $=10.8$ $\mathrm{ms}$ vs. right $\left.\mathrm{VF}=7.7 \mathrm{~ms}: F(1,46)=0.218, p=0.643, \eta_{p}^{2}=0.005\right)$. Therefore, these results of the post hoc analyses supported the possibility that differences in the degree of bi-hemispheric involvement by handedness lead to the application of different cognitive strategies.

Further research is needed to increase the generalizability of the idea that handedness determines the application of different unintentional cognitive strategies. Specifically, it is necessary to investigate this effect using stimulus-response (S-R) compatibility paradigms with feature overlap between the response set and irrelevant stimulus dimensions other than the Simon task (Type 3 S-R compatibility in the dimensional overlap model's taxonomy of Kornblum, Hasbroucq, \& Osman) [33]. It is worth noting that manipulating the PCs in a given Simon task block creates a situation in which response-button presses ipsilateral to the target location side increases (or decreases) under conditions in which congruent trials are more (or less) frequent. In other words, specific task-relevant information about a target presented in the lateral VF is strongly associated with the specific button press side. Therefore, the block-wise PC effect observed in the Simon task is more closely related to stimulus-response contingency (learning ac- 
count of the block-wise PC effect) than observed in the Flanker task or the Stroop task, which have characteristics of stimulus-stimulus compatibility (Type $4 \mathrm{~S}-\mathrm{R}$ compatibility in the taxonomy of Kornblum et al.) [33].

In conclusion, this study demonstrated the left-hander's advantage for the block-wise PC effect in the Simon task, suggesting that left- and right-handers unintentionally apply different cognitive strategies in response to conflict resolution experiences. These findings supported the stability-flexibility view of handedness in cognitive control. Furthermore, the present findings suggest that future cognitive studies, as well as laterality studies, should take into account the potential cognitive strategy biases depending on handedness.

\section{Funding}

This research was supported in part by a program grant from the Japan Society for the Promotion of Science KAKENHI Grant Number JP15K04198 to Kazuhito Yoshizaki. The study was approved by the Institutional Ethics Committee of Aichi Shukutoku University (No. 2017-01-r02) and was consistent with the 2008 Declaration of Helsinki on experiments using human subjects.

\section{Conflicts of Interest}

The authors have no competing interests with this study's findings. The data that support the findings of this study are openly available in "dataset_Dominant hand in forming" at http://kazu01.sakura.ne.jp/cogneuro/data-set-based-on-das/.

\section{References}

[1] Guadalupe, T., Willems, R.M., Zwiers, M.P., Vasquez, A.A., Hoogman, M., Hagoort, P., Francks, C., et al. (2014) Differences in Cerebral Cortical Anatomy of Left- and Right-Handers. Frontiers in Psychology, 5, Article No. 261.

https://doi.org/10.3389/fpsyg.2014.00261

[2] Badzakova-Trajkov, G., Häberling, I.S., Roberts, R.P. and Corballis, M.C. (2010) Cerebral Asymmetries: Complementary and Independent Processes. PLOS ONE, 5, e9682. https://doi.org/10.1371/journal.pone.0009682

[3] Hécaen, H., De Agostini, M. and Monzon-Montes, A. (1981) Cerebral Organization in Left-Handers. Brain \& Language, 12, 261-284. https://doi.org/10.1016/0093-934X(81)90018-3

[4] Willems, R.M., Peelen, M.V. and Hagoort, P. (2010) Cerebral Lateralization of Face-Selective and Body-Selective Visual Areas Depends on Handedness. Cerebral Cortex, 20, 1719-1725. https://doi.org/10.1093/cercor/bhp234

[5] Gunstad, J., Spitznagel, M.B., Luyster, F., Cohen, R.A. and Paul, R.H. (2007) Handedness and Cognition across the Healthy Lifespan. International Journal of Neuroscience, 117, 477-485. https://doi.org/10.1080/00207450600773483

[6] Ocklenburg, S. and Güntürkün, O. (2017) The Lateralized Brain: The Neuroscience and Evolution of Hemispheric Asymmetries. Academic Press, London. https://doi.org/10.1016/B978-0-12-803452-1.00009-6

[7] Goschke, T. and Bolte, A. (2014) Emotional Modulation of Control Dilemmas: The Role of Positive Affect, Reward, and Dopamine in Cognitive Stability and Flexibili- 
ty. Neuropsychologia, 62, 403-423.

https://doi.org/10.1016/j.neuropsychologia.2014.07.015

[8] Hommel, B. and Colzato, L.S. (2017) The Social Transmission of Metacontrol Policies: Mechanisms Underlying the Interpersonal Transfer of Persistence and Flexibility. Neuroscience and Biobehavioral Reviews, 8, 43-58. https://doi.org/10.1016/j.neubiorev.2017.01.009

[9] Serrien, D.J. and O'Regan, L. (2019) Stability and Flexibility in Cognitive Control: Interindividual Dynamics and Task Context Processing. PLoS ONE, 14, e0219397. https://doi.org/10.1371/journal.pone.0219397

[10] Sontam, V., Christman, S.D. and Jasper, J.D. (2009) Individual Differences in Semantic Switching Flexibility: Effects of Handedness. Journal of the International Neuropsychological Society, 15, 1023-1027. https://doi.org/10.1017/S1355617709990440

[11] Fitts, P.M. and Seeger, C.M. (1953) S-R Compatibility: Spatial Characteristics of Stimulus and Response Codes. Journal of Experimental Psychology, 46, 199-210. https://doi.org/10.1037/h0062827

[12] Eriksen, B.A. and Eriksen, C.W. (1974) Effects of Noise Letters upon the Identification of a Target Letter in a Nonsearch Task. Perception \& Psychophysics, 16, 143-149. https://doi.org/10.3758/BF03203267

[13] Stroop, J.R. (1935) Studies of Interference in Serial Verbal Reactions. Journal of EXperimental Psychology, 18, 643-662. https://doi.org/10.1037/h0054651

[14] Simon, J.R. (1990) The Effects of an Irrelevant Directional Cue on Human Information Processing. In: Proctor, R.W. and Reeve, T.G., Eds., Stimulus-Response Compatibility: An Integrated Perspective, North-Holland, Amsterdam, 31-86. https://doi.org/10.1016/S0166-4115(08)61218-2

[15] Egner, T. (2007) Congruency Sequence Effects and Cognitive Control. Cognitive, Affective and Behavioral Neuroscience, 7, 380-390. https://doi.org/10.3758/CABN.7.4.380

[16] Gratton, G., Coles, M.G.H. and Donchin, E. (1992) Optimizing the Use of Information: Strategic Control of Activation of Responses. Journal of Experimental Psychology: General, 121, 480-506. https://doi.org/10.1037/0096-3445.121.4.480

[17] Kuratomi, K. and Yoshizaki, K. (2016) Flexible Adjustments of Visual Selectivity in a Flanker Task. Journal of Cognitive Psychology, 28, 462-473.

https://doi.org/10.1080/20445911.2015.1135157

[18] Logan, G.D. and Zbrodoff, N.J. (1979) When It Helps to Be Misled: Facilitative Effects of Increasing the Frequency of Conflicting Stimuli in a Stroop-Like Task. Memory \& Cognition, 7, 166-174. https://doi.org/10.3758/BF03197535

[19] Torres-Quesada, M., Lupiáñez, J., Milliken, B. and Funes, M.J. (2014) Gradual Proportion Congruent Effects in the Absence of Sequential Congruent Effects. Acta Psychologica, 149, 78-86. https://doi.org/10.1016/j.actpsy.2014.03.006

[20] Bugg, J.M. and Crump, M.J.C. (2012) In support of a Distinction between Voluntary and Stimulus-Driven Control: A Review of the Literature on Proportion Congruent Effects. Frontiers in Psychology, 3, Article No. 367. https://doi.org/10.3389/fpsyg.2012.00367

[21] Abrahamse, E., Braem, S., Notebeart, W. and Verguts, T. (2016) Grounding Cognitive Control in Associative Learning. Psychological Bulletin, 142, 693-728. https://doi.org/10.1037/bul0000047

[22] Ghinescu, R., Schachtman, T.R., Stadler, M.A., Fabiani, M. and Gratton, G. (2010) Strategic Behavior without Awareness? Effects of Implicit Learning in the Eriksen 
Flanker Paradigm. Memory \& Cognition, 38, 197-205.

https://doi.org/10.3758/MC.38.2.197

[23] Kuratomi, K. and Yoshizaki, K. (2012) Explicit Instruction Does Not Modulate BlockWise Conflict Adaptation. The 24th Annual Convention, Association for Psychological Science, Chicago, 24-27 May 2012.

[24] Abrahamse, E., Duthoo, W., Notebeart, W. and Risko, E.F. (2013) Attention Modulation by Proportion Congruency: The Asymmetrical List Shifting Effect. Journal of Experimental Psychology: Learning, Memory, and Cognition, 39, 1552-1562. https://doi.org/10.1037/a0032426

[25] Schmidt, J.R. (2012) Human Contingency Learning. In: Seal, N.M., Ed., Encyclopedia of the Sciences of Learning, Springer, New York, 1455-1456.

https://doi.org/10.1007/978-1-4419-1428-6 646

[26] Schmidt, J.R. (2013) Questioning Conflict Adaptation: Proportion Congruent and Gratton Effects Reconsidered. Psychological Bulletin \& Review, 20, 615-630. https://doi.org/10.3758/s13423-012-0373-0

[27] Schmidt, J.R. and Besner, D. (2008) The Stroop Effect: Why Proportion Congruent Has Nothing to Do with Congruency and Everything to Do with Contingency. Journal of Experimental Psychology: Learning, Memory, and Cognition, 34, 514-523. https://doi.org/10.1037/0278-7393.34.3.514

[28] Okubo, M., Suzuki, H. and Nicholls, M.E.R. (2014) A Japanese Version of the Flanders Handedness Questionnaire. Japanese Journal of Psychology, 85, 474-481.

https://doi.org/10.4992/jipsy.85.13235

[29] Cherbuin, N. and Brinkman, C. (2006) Hemispheric Interactions Are Different in Left-Handed Individuals. Neuropsychology, 20, 700-707. https://doi.org/10.1037/0894-4105.20.6.700

[30] Cowell, P.E. and Gurd, J. (2018) Handedness and the Corpus Callosum: A Review and Further Analyses of Discordant Twins. Neuroscience, 388, 57-68. https://doi.org/10.1016/j.neuroscience.2018.06.017

[31] Rosch, R.E., Cowell, P.E. and Gurd, J.M. (2018) Cerebellar Asymmetry and Cortical Connectivity in Monozygotic Twins with Discordant Handedness. Cerebellum, 17, 191-203. https://doi.org/10.1007/s12311-017-0889-y

[32] Johnstone, L.T., Karlsson, E.M. and Carey, D.P. (2021) Left-Handers Are Less Lateralized than Right-Handers for both Left and Right Hemispheric Functions. Cerebral Cortex, 31, 3780-3787. https://doi.org/10.1093/cercor/bhab048

[33] Kornblum, S., Hasbroucq, T. and Osman, A. (1990) Dimensional Overlap: Cognitive Basis for Stimulus-Response Compatibility: A Model and Taxonomy. Psychological Review, 97, 253-270. https://doi.org/10.1037/0033-295X.97.2.253 